\title{
Experimental evaluation in wireless communications
}

\author{
José A. García-Naya ${ }^{*}{ }^{*}$, Robert Heath ${ }^{2}$, Florian Kaltenberger ${ }^{3}$, Markus Rupp ${ }^{4}$ and Javier Vía ${ }^{5}$
}

\begin{abstract}
This editorial sums up relevant topics on the assessment of wireless communication systems covered by the especial issue entitled "Experimental Evaluation in Wireless Communications." The topics include practical aspects on the implementation of distributed asynchronous non-linear kernel methods over wireless sensor networks; localization methods based on the exploitation of radio-frequency identification (RFID) wireless sensors and cellular networks or on sparsity approximations; channel sounding and assessment of broadband orthogonal frequency-division multiplexing (OFDM)-based wireless systems in high-speed vehicular communications; coexistence analysis of femtocell-based and outdoor-to-indoor systems; techniques for peak-to-average power ratio (PAPR) reduction; new solutions for baseband and radio frequency (RF) hardware impairments in full-duplex wireless systems; and, finally, suitability of interference alignment for broadband indoor wireless communications.
\end{abstract}

\section{Special issue motivation and overview}

The benefits of experimentation are not always realized in research on signal processing for wireless communication systems. In wireless systems, many abstractions are made to facilitate analysis. With unknown channels and realistic hardware, experimentation is a crucial means towards understanding the real-world viability of new ideas. Furthermore, during such experimental work, often new research questions arise as it may turn out that not all aspects have been sufficiently modeled.

This special issue brings together research results obtained through experimental evaluation and measurements. The applications embrace cellular transmissions such as Long-Term Evolution Advanced (LTE-A), interference alignment techniques, local area networks, vehicular networks, wireless sensor networks, localization, and other wireless transmission systems.

After a rigorous peer review process, the following 12 articles were selected for publication out of 42 submissions. All papers received at least three high quality reviews. We appreciate the careful and methodical

*Correspondence: jagarcia@udc.es

'Department of Electronics and Systems, University of A Coruña, Campus de Elviña s/n, 15071 A Coruña, Spain

Full list of author information is available at the end of the article reviews. We would like to thank all the authors for submitting their work to our special issue and also all the reviewers for their efforts.

The published articles were grouped according to the topics addressed, and they are briefly introduced in the following.

Wireless sensor networks received a great attention in this special issue. Implementation aspects of distributed and asynchronous non-linear kernel methods were addressed in [1]. For their evaluations, they employed MICAz motes running TinyOS 2.1.1, and the practical aspects considered were related to matrix and floating computations, acknowledgment mechanisms, and data loss. On the other hand, wireless sensor networks are typically employed in localization applications. In [2], a target sensor was located using techniques inspired in the recent advances on the so-called sparse approximation. This way, the localization problem was defined as a block-sparse signal recovery problem in the discrete spatial domain. More specifically, the authors proposed received signal strength indication (RSS)-fingerprinting algorithms that were evaluated both via simulation and on-field experiments. Finally, [3] also addressed the problem of localization in indoor scenarios by simultaneously combining the information provided by radio-frequency identification (RFID) sensors and cellular networks.

Another topic that brought a lot of attention was the assessment of broadband wireless communications 
in mobile scenarios. Channel capacity of multiple-input multiple-output (MIMO) systems based on orthogonal frequency-division multiplexing (OFDM) in vehicular-toinfrastructure environments at $920 \mathrm{MHz}$ was investigated in [4]. The authors also took into account the effects of channel estimation errors in the three different wireless systems considered in the measurement campaigns: IEEE 802.11p, Long-Term Evolution (LTE), and Digital Video Broadcasting (DVB)-T2. Unfortunately, experimental evaluations of wireless systems in scenarios with high mobility are expensive and complex and require infrastructures and vehicles that are not easily available (e.g., high-speed trains). To overcome this problem, a technique to induce effects caused by highly time-varying channels on OFDM signals while conducting measurements at low speeds was proposed in [5]. Such a technique was validated by means of LTE measurements at different velocities in a controlled measurement environment and also using computer simulations. Using the aforementioned technique, the effects of inter-carrier interference in the channel estimation process and in the resulting achievable throughput with respect to the feedback delay were investigated in [6]. For the experimental evaluation, the authors considered the downlink of IEEE 802.16e, also known as Mobile WiMAX, in high-mobility scenarios. Outdoor-tooutdoor and outdoor-to-indoor propagation conditions combined with single- and multiple antenna transmissions were evaluated. Finally, [7] reported vehicular channel measurements in the frequency band of $5.8 \mathrm{GHz}$ for the IEEE 802.11p standard. Experiments for both intravehicle and out-of-vehicle environments were carried out, and the obtained results strongly vouch for the suitability of the 802.11p standard for in-car as well as out-of-car wireless applications in stationary environments.

The next topic addressed in the special issue is the coexistence between indoor LTE femtocells and outdoorto-indoor DVB-T2-Lite in a shared frequency band, which was assessed in [8].

A well-known undesired property of OFDM is its strong peak-to-average power ratio (PAPR). Techniques to overcome this problem were considered in two articles of the special issue. The first one [9] focused on a fieldprogrammable gate array (FPGA) implementation of the peak cancellation technique, which is known as the simplest method for PAPR reduction. The design issue of effective peak-canceling pulses under the constraint on the out-of-band emission is addressed. In order to reduce its hardware complexity, a novel approach for generating peak-canceling pulses was also presented. The second article [10] proposed a hybrid peak-windowing scheme that selects the optimum PAPR reduction scheme according to the PAPR pattern. The performance of the proposed technique is evaluated using an LTE testbed considering also the power consumption.
Hardware imperfections can significantly reduce the performance of full-duplex wireless systems, hence making it challenging to fully suppress self-interference. In [11], a measurement-based approach to experimentally identify and isolate these hardware imperfections leading to residual self-interference in full-duplex nodes is followed. Measurement results showed the important role of images arising from in-phase and quadrature (IQ) imbalance in the transmitter and receiver mixers as well as baseband non-linearities in the digital-to-analog converters (DACs), which can introduce strong harmonic components in the transmitted signal. A corresponding general mathematical model to suppress these components of the self-interference signal arising from the hardware nonidealities was developed from the observations and measurements. Results from a 10-MHz bandwidth full-duplex system operating at $2.48 \mathrm{GHz}$ showed that up to $13 \mathrm{~dB}$ additional suppression, relative to state-of-the-art implementations, can be achieved by jointly compensating for IQ imbalance and DAC non-linearities.

Interference management became a key issue in the design of wireless systems. Traditional orthogonal multiple access schemes divide bandwidth and/or time resources among users, and the individual data rates decrease with the size of the network. interference alignment (IA) has been recently proposed as a promising interference management technique. An experimental study on the performance of spatial IA in indoor wireless local area network scenarios that use OFDM according to the IEEE 802.11a standard is presented in [12]. Experiments have been carried out using a wireless network testbed capable of implementing a 3-user MIMO interference channel and considering IA decoding schemes designed according to distinct criteria (e.g., zero-forcing or MaxSINR). The performance of IA in terms of bit error rate measurement-based results was also compared to different time-division multiple access transmission schemes, including single- and multiple antenna systems transmitting over the dominant mode of the MIMO channel. The results indicated that spatial IA is suitable for practical indoor scenarios in which wireless channels often exhibit relatively large coherence times.

\section{Acknowledgements \\ The editors would like to thank the authors for their interest in this special issue, their cooperation, and their outstanding contributions, which are reflected in this issue. This special issue would have not been possible without the support of reviewers, and we also thank them. We thank our Editor-in-Chief, Prof. Luc Vandendorpe. Finally, we thank the entire Editorial Office Staff for their dedication and effort devoted to this special issue.}

Authors' contributions

All authors read and approved the final manuscript.

Competing interests

The authors declare that they have no competing interests. 


\section{Publisher's Note}

Springer Nature remains neutral with regard to jurisdictional claims in published maps and institutional affiliations.

\section{Author details}

${ }^{1}$ Department of Electronics and Systems, University of A Coruña, Campus de Elviña s/n, 15071 A Coruña, Spain. ${ }^{2}$ Department of Electrical and Computer Engineering, The University of Texas at Austin, Austin, TX 78712, USA.

${ }^{3}$ EURECOM, Sophia Antipolis, 06410 Biot, France. ${ }^{4}$ Institute of

Telecommunications, TU Wien, Gusshausstrasse 25/389, A-1040 Vienna, Austria. ${ }^{5}$ Department of Communications Engineering, University of Cantabria, 39005 Santander, Spain.

Received: 20 March 2017 Accepted: 20 March 2017

Published online: 28 March 2017

\section{References}

1. JA Garrido-Castellano, JJ Murillo-Fuentes, On the implementation of distributed asynchronous non-linear kernel methods over wireless sensor networks. EURASIP J. Wirel. Commun. Netw. 2015(1), 171 (2015). doi:10.1186/s13638-015-0382-6

2. A Bay, D Carrera, SM Fosson, P Fragneto, M Grella, C Ravazzi, E Magli, Blocksparsity-based localization in wireless sensor networks. EURASIP J. Wirel. Commun. Netw. 2015(1), 182 (2015). doi:10.1186/s13638-015-0410-6

3. A Aguilar-Garcia, S Fortes, E Colin, R Barco, Enhancing RFID indoor localization with cellular technologies. EURASIP J. Wirel. Commun. Netw. 2015(1), 219 (2015). doi:10.1186/s13638-015-0444-9

4. OJ Onubogu, K Ziri-Castro, D Jayalath, H Suzuki, Experimental evaluation of the performance of $2 \times 2$ MIMO-OFDM for vehicle-to-infrastructure communications. EURASIP J. Wirel. Commun. Netw. 2015(1), 183 (2015). doi:10.1186/s13638-015-0411-5

5. J Rodríguez-Piñeiro, M Lerch, JA García-Naya, S Caban, M Rupp, L Castedo, Emulating extreme velocities of mobile LTE receivers in the downlink. EURASIP J. Wirel. Commun. Netw. 2015(1), 106 (2015). doi:10.1186/s13638-015-0343-0

6. P Suárez-Casal, J Rodríguez-Piñeiro, JA García-Naya, L Castedo, Experimental evaluation of the WiMAX downlink physical layer in high-mobility scenarios. EURASIP J. Wirel. Commun. Netw. 2015(1), 109 (2015). doi:10.1186/s13638-015-0339-9

7. P Kukolev, A Chandra, T Mikulášek, A Prokeš, T Zemen, CF Mecklenbräuker, In-vehicle channel sounding in the 5.8-GHz band. EURASIP J. Wirel. Commun. Netw. 2015(1), 57 (2015). doi:10.1186/s13638-015-0273-x

8. L Polak, L Klozar, O Kaller, J Sebesta, M Slanina, T Kratochvil, Study of coexistence between indoor LTE femtocell and outdoor-to-indoor DVB-T2-Lite reception in a shared frequency band. EURASIP J. Wirel. Commun. Netw. 2015(1), 114 (2015). doi:10.1186/s13638-015-0338-x

9. J Song, H Ochiai, A low-complexity peak cancellation scheme and its FPGA implementation for peak-to-average power ratio reduction. EURASIP J. Wirel. Commun. Netw. 2015(1), 85 (2015). doi:10.1186/s13638-015-0319-0

10. D Kim, S An, Experimental analysis of PAPR reduction technique using hybrid peak windowing in LTE system. EURASIP J. Wirel. Commun. Netw. 2015(1), 75 (2015). doi:10.1186/s13638-015-0282-9

11. A Balatsoukas-Stimming, AC Austin, P Belanovic, A Burg, Baseband and RF hardware impairments in full-duplex wireless systems: experimental characterisation and suppression. EURASIP J. Wirel. Commun. Netw. 2015(1), 142 (2015). doi:10.1186/s13638-015-0350-1

12. C Lameiro, Ó González, JA García-Naya, I Santamaría, L Castedo, Experimental evaluation of interference alignment for broadband WLAN systems. EURASIP J. Wirel. Commun. Netw. 2015(1), 180 (2015). doi:10.1186/s13638-015-0409-z

\section{Submit your manuscript to a SpringerOpen ${ }^{\circ}$ journal and benefit from:}

- Convenient online submission

- Rigorous peer review

- Immediate publication on acceptance

- Open access: articles freely available online

- High visibility within the field

- Retaining the copyright to your article

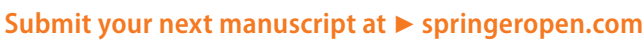

